\title{
Improving the management of patients at risk of haemorrhagic stroke
}

W

hen a patient presents

with an unruptured brain

arteriovenous malformation

(AVM) or unruptured intracranial aneurysm, the attending clinician is faced with the difficult decision of whether to undertake interventional therapy such as neurosurgery-and risk treatmentassociated morbidity and mortality - to avoid a haemorrhagic event that might never occur. Two studies published in The Lancet and The Lancet Neurology could help to address this contentious issue.

Detection of cerebrovascular abnormalities has improved substantially in the past few decades owing to advances in imaging techniques. Previous studies suggested that annual rupture rates of AVMs could be as low as $1 \%$ and, moreover, first haemorrhagic events in this syndrome tend to be mild. In this context of increased identification of individuals with unruptured AVMs and potentially low risks of a serious haemorrhagic events, A Randomized trial of Unruptured Brain AVMs (ARUBA) was conducted to determine how best to manage such individuals.

Trial participants were prospectively enrolled from 39 clinical sites in nine countries, and were randomly assigned to receive medical management alone or medical management plus interventional therapy (neurosurgery, embolization and/or stereotactic radiotherapy). The investigators had planned to follow 800 patients for 5 years, but the trial was halted early-after follow-up of 223 individuals for a mean time of 33 months-owing to superiority of the medical management group at an interim analysis.

114 patients were assigned to the interventional therapy group and 109 were assigned to medical management alone. At the interim analysis, 35 (30.7\%) patients in the interventional therapy group had reached the primary end point of death or symptomatic stroke, compared with only $11(10.1 \%)$ patients in the medical

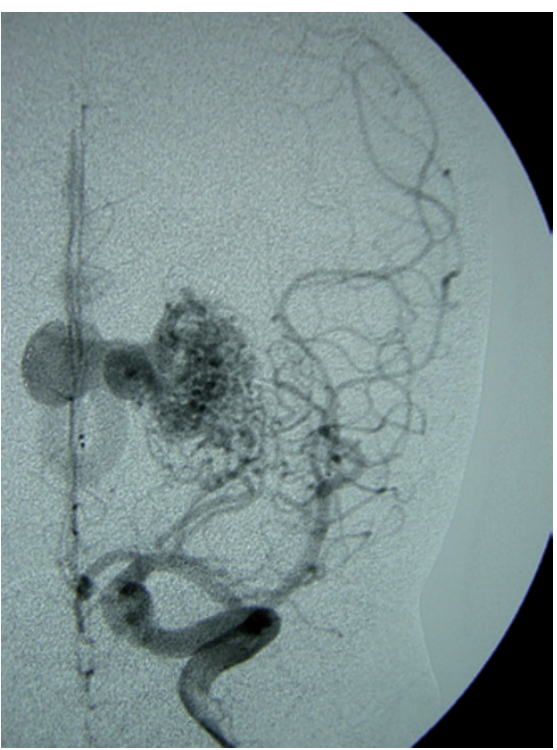

Cerebral angiogram showing an arteriovenous malformation. Image courtesy of C. Stapf.

management group. "The risk of death and symptomatic stroke is almost four times lower in patients managed without interventional therapy," says ARUBA investigator Christian Stapf. "Also, after 3 years the risk of death and neurological disability is three times lower for patients managed without intervention."

The trial confirmed the low rate of spontaneous rupture of AVMs in patients who did not receive interventional therapy (2.2\% per year).

"This is the first ever randomized controlled trial of any intervention for brain AVMs, and as such it is hugely welcome," says Charles Warlow, Emeritus Professor of Medical Neurology at the Centre For Clinical Brain Sciences, Edinburgh, UK, who was not involved in the study. "However, the result still leaves us uncertain what to do for the best in the long term because the trial was stopped after a mere 33 months. Patients with AVMs are young or middle-aged and need to know how things will go over 33 years!"

Stapf says that they are seeking funding to continue the observational phase for at least 5 more years to establish whether the disparities in clinical outcome between the two treatment approaches will persist over time.

In the second study, Jacoba Greving and colleagues sought to devise a tool to enable estimation of risk of rupture of an intracranial aneurysm at the individual patient level. Intracranial aneursyms are common in the general population, at about $3 \%$, and rupture can lead to subarachnoid haemorrhage-a stroke subtype with high case fatality. However, ruptures are uncommon and, as in the case of AVMs, neurosurgical intervention can prove more harmful than beneficial.

The researchers conducted a systematic review and pooled analysis of data from 8,382 individuals with an unruptured intracranial aneurysm from six prospective cohort studies. Aneurysm rupture occured in 230 patients, and 1-year and 5-year rupture risks were 1.4\% and $3.4 \%$, respectively.

Greving et al. identified six key predictors of aneurysm rupture: age, hypertension, history of subarachnoid haemorrhage, aneurysm size, aneurysm location, and geographical region. The researchers used their findings to develop a risk score, termed PHASES, which can be used to grade patients on each of the predictors and, thereby, determine an individual patient's risk of aneurysm rupture. This tool could help to inform treatment decisions and individualize the management of intracranial aneurysms.

Katie Kingwell 